\title{
Broadband Cooling Spectra of Hot Electrons and Holes in PbSe Quantum Dots
}

\author{
Frank C. M. Spoor, ${ }^{\dagger}$ Stanko Tomić, ${ }^{\dagger}$ Arjan J. Houtepen, ${ }^{*}{ }^{\dagger}$ and Laurens D. A. Siebbeles ${ }^{*} \dagger$ (1)
}

${ }^{\dagger}$ Optoelectronic Materials Section, Department of Chemical Engineering, Delft University of Technology, Van der Maasweg 9, 2629 HZ Delft, The Netherlands

${ }^{\ddagger}$ Joule Physics Laboratory, School of Computing, Science and Engineering, University of Salford, Manchester M5 4WT, United Kingdom

\section{Supporting Information}

ABSTRACT: Understanding cooling of hot charge carriers in semiconductor quantum dots (QDs) is of fundamental interest and useful to enhance the performance of QDs in photovoltaics. We study electron and hole cooling dynamics in PbSe QDs up to high energies where carrier multiplication occurs. We characterize distinct cooling steps of hot electrons and holes and build up a broadband cooling spectrum for both charge carriers. Cooling of electrons is slower than of holes. At energies near the band gap we find cooling times between successive electronic energy levels in the order of $0.5 \mathrm{ps}$. We argue that here the large spacing between successive electronic energy levels requires cooling to occur by energy transfer to vibrational modes of ligand molecules or phonon modes associated with the QD surface. At high excess energy the energy loss rate of electrons is 1-5 $\mathrm{eV} / \mathrm{ps}$ and exceeds $8 \mathrm{eV} / \mathrm{ps}$ for holes. Here charge carrier cooling can be understood in terms of emission of LO phonons with a higher density-of-states in the valence band than the conduction band. The complete mapping of the broadband cooling spectrum for both charge carriers in PbSe QDs is a big step toward understanding and controlling the cooling of hot charge carriers in colloidal QDs.

KEYWORDS: quantum dot, nanocrystal, carrier cooling, transient absorption spectroscopy, electronic structure, carrier dynamics
$\mathrm{C}$ olloidal semiconductor quantum dots (QDs) are of interest because of the possibility of tuning their optical and electronic properties by variation of composition and size. In combination with their solutionprocessability this offers promising prospects for application in devices such as field-effect transistors, light-emitting diodes, photodetectors, and solar cells. ${ }^{1} \mathrm{PbSe} \mathrm{QDs}$ receive much attention due to their exceptionally strong quantum confinement properties, and in particular because of the occurrence of carrier multiplication $(\mathrm{CM}){ }^{2-6} \mathrm{CM}$ is a process in which one sufficiently energetic photon excites two or more electrons across the band gap. In this way the power conversion efficiency of a solar cell can be enhanced above the ShockleyQueisser limit. CM occurs in competition with cooling of initially energetic electrons or holes via $\mathrm{LO}$ phonon emission or other relaxation channels. ${ }^{7-9}$

Until now, studies on charge carrier cooling in PbSe QDs involved low photoexcitation energies close to the band gap. ${ }^{10-13}$ Here, the distance between adjacent electronic energy levels is typically several LO phonon energies, requiring simultaneous emission of multiple phonons for a charge carrier to cool from one level to the one below. This is referred to as the "phonon bottleneck" and was originally suggested to increase the $\mathrm{CM}$ efficiency of nanocrystals over bulk. ${ }^{14,15}$ However, clear evidence of the phonon bottleneck has not been found in PbSe QDs. ${ }^{12,16}$ Near the band edge other cooling channels can become dominant, such as trapping at defects, energy transfer to vibrational modes of ligand molecules, or surface related phonon modes. ${ }^{13,17-21}$ In agreement with this, it was found that electron cooling near the band edge in CdSe QDs is slowed down several orders of magnitude by decoupling the electron from the surface, so that it could relax only through emission of $\mathrm{LO}$ phonons. ${ }^{22,23}$

The competition between $\mathrm{CM}$ and other relaxation channels takes place at higher energies where an electron or a hole has an excess energy that is at least equal to the band gap. At such high excess energy the electronic structure of $\mathrm{PbSe} \mathrm{QDs}$ approaches that of bulk $\mathrm{PbSe}$ and $\mathrm{LO}$ phonon emission will not be restricted by the availability of electronic energy levels at a spacing equal to the phonon energy. Currently, little is known of cooling at high charge carrier excess energy, except that it is

Received: April 11, 2017

Accepted: May 30, 2017

Published: May 30, 2017 
very fast. $^{24-28}$ In addition, hot carrier cooling in QDs is usually described as a single cooling time. ${ }^{12,29-31}$ We stress however that each transition of a charge carrier from an energy level to a level of lower energy is a discrete quantum mechanical transition with its own characteristic time constant. Hence carrier cooling times are a function of energy and should ideally be described by a cooling spectrum, separate for electrons and holes.

In this work, we determine cooling times of charge carriers in $\mathrm{PbSe}$ QDs between discrete electronic states near the band edge, and at higher excess energy where the electronic states form a quasi-continuum with a character close to that of bulk PbSe. We build up a broadband cooling spectrum for both charge carriers and find that holes cool faster than electrons, in agreement with our earlier work. ${ }^{32}$ Strikingly, we can resolve five discrete cooling steps (three for electrons and two for holes) near the band edge before the onset of the quasicontinuum. We argue that at lower energies charge carrier cooling involves energy transfer to vibrational modes associated with the surface of the QD. At high carrier excess energy cooling can be explained in terms of emission of LO phonons, which we verify by $\boldsymbol{k} \cdot \boldsymbol{p}$ calculations. We show that we expect an asymmetric density-of-states (DOS) with a denser valence band than conduction band to account for the differences in cooling times of electrons and holes.

\section{RESULTS AND DISCUSSION}

Measurements and Modeling of Electron and Hole Cooling Dynamics. PbSe QDs with a diameter of $3.9 \mathrm{~nm}$ and band gap of $0.93 \mathrm{eV}$ were synthesized via the method by Steckel et al., ${ }^{33}$ washed twice, and dispersed in toluene (see Methods). The QD dispersion was investigated using transient absorption (TA) spectroscopy with a time resolution of $\sim 0.15$ ps and a probe spectral range of 375-1600 nm (see Methods). In all measurements discussed below the pump laser fluence was sufficiently low that on average much less than one photon per QD was absorbed $\left(\left\langle N_{\mathrm{abs}}\right\rangle<0.15\right)$. We show in Figure 1a the hyperspectral TA image that results from photoexcitation by a pump laser pulse with photon energy of $3.35 \mathrm{eV}(370 \mathrm{~nm})$, which is high above the band gap. At this photoexcitation energy CM takes place and Auger recombination of multiexcitons can be observed in the first tens of picoseconds. After that only single cold excitons are left and no further decay is observed in the considered time window of $2.5 \mathrm{~ns}$, since the single exciton lifetime in PbSe QDs is several hundreds of nanoseconds. ${ }^{13,34}$ The main aim of this work is to study charge carrier cooling, which can be observed in the first few picoseconds after photoexcitation. To highlight the spectral features of charge carrier cooling, we show spectral slices at short (averaged 0.5-1.0 ps) and long (averaged 0.5-2.5 ns) pump-probe delay in Figure 1b, together with the ground-state absorption spectrum. Vertical dashed lines are added to indicate the position of several optical transitions as identified in the past (labeled $S$ for the $1 S_{e}-1 S_{h}$ transition, $P$ for $1 P_{h}-1 P_{e}, D$ for $1 D_{h}-1 D_{e}$, and $\Sigma$ for quantum confined transitions around the $\Sigma$-point in the bulk Brillouin Zone). ${ }^{35}$

The spectral features at long pump-probe delay in Figure $1 \mathrm{~b}$ are due to single cold $1 S_{\mathrm{h}}-1 \mathrm{~S}_{\mathrm{e}}$ excitons, as discussed in our previous work. ${ }^{32}$ The negative TA signal near $1330 \mathrm{~nm}$ is due to ground state bleaching and stimulated emission resulting from $1 S_{\mathrm{h}}-1 S_{\mathrm{e}}$ excitons. The negative TA signal at $400-500 \mathrm{~nm}$ results from blocking of transitions to the second conduction or valence band by the presence of either a hole in the valence

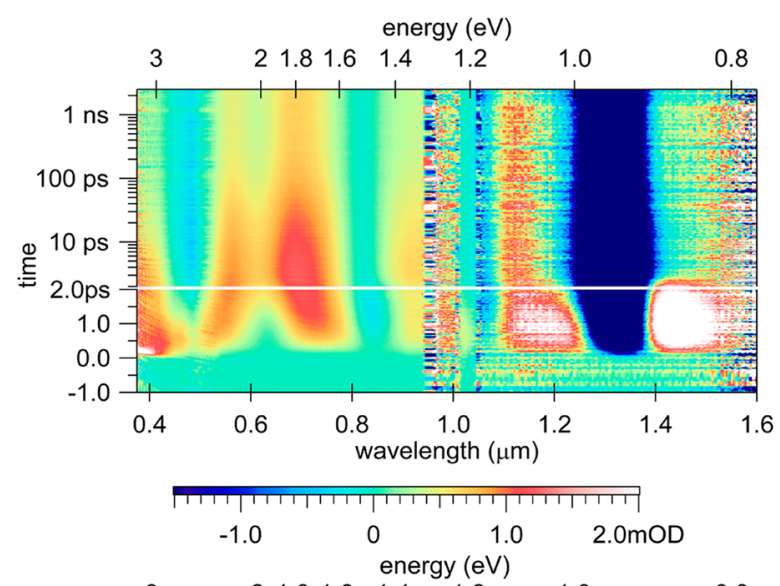

(b)

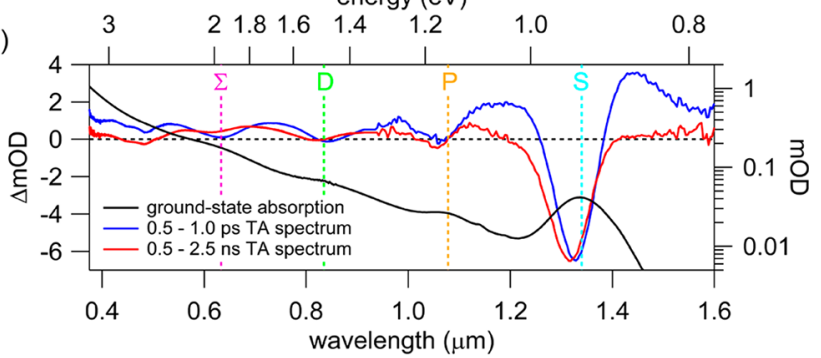

Figure 1. (a) Hyperspectral TA image for $3.9 \mathrm{~nm}$ PbSe QDs photoexcited by a pump laser pulse with photon energy of $3.35 \mathrm{eV}$ $(370 \mathrm{~nm})$. The TA image is corrected for dispersion of the probe light (see Methods). (b) Spectral slices taken at short (averaged 0.5-1.0 ps) and long (averaged 0.5-2.5 ns) pump-probe delay, together with the ground-state absorption spectrum and vertical dashed lines to indicate several optical transitions.

band $\left(1 S_{h}\right)$ or an electron in the conduction band $\left(1 S_{e}\right){ }^{32}$ Between these bleach features, the transient absorption spectrum is caused by a red-shift of the ground-state absorption spectrum due to biexciton interactions and possible intraband absorption. ${ }^{36}$

The negative TA signals are very different at short pumpprobe delay when hot excitons are cooling down to the band edge. The band edge bleach is already present, but accompanied by a photoinduced absorption (PA) signal below the band gap. Moreover, a PA contribution shifts the TA signal at $400-500 \mathrm{~nm}$ toward positive values. These PA signals result from biexciton interactions caused by hot excitons and largely disappear when cooling has completed. ${ }^{37,38}$

In literature, charge carrier cooling is often studied by considering single wavelength TA signals at the $1 \mathrm{~S}_{\mathrm{h}}-1 \mathrm{~S}_{\mathrm{e}}$ and $1 \mathrm{P}_{\mathrm{h}}-1 \mathrm{P}_{\mathrm{e}}$ transitions. ${ }^{12,13,22,26,39}$ This however does not take into account the PA signal caused by biexciton interactions near these transitions. ${ }^{29,40,41}$ As cooling takes place, the PA signal decreases and the band edge bleach increases, since fewer hot excitons are present and more excitons have arrived at the band edge. To quantitatively describe the band edge bleach while correcting for biexciton interactions, one can either fit or integrate. $^{29,40}$ We choose here to fit because it alleviates problems with a coherent artifact and boundaries, but we show in Figure S1 of the Supporting Information that identical results can be obtained. We first fit a Gaussian function to the $1 S_{h}-1 S_{e}$ transition peak in the ground-state absorption spectrum $A$ as a function of photon energy. We then assume that the bleach of the transition is directly proportional to the number of $1 S_{h}-1 S_{e}$ excitons present. The corresponding absorption peak of the 
excited state absorption spectrum $A^{*}$ at time $t$ after the pump laser pulse is then given by

$$
\begin{aligned}
& A^{*}=A+\Delta A=(1-f(t)) C \mathrm{e}^{-\left(E-E_{0}\right)^{2} / 2 \sigma^{2}} \\
& \quad+f(t) \frac{3}{4} \mathrm{Ce}^{-\left(E-\left(E_{0}-\delta E(t)\right)\right)^{2} / 2 \sigma^{2}}
\end{aligned}
$$

where the parameters $C, E_{0}$, and $\sigma$ are obtained from the Gaussian fit to the ground-state absorption spectrum. The function $f(t)$ is the sum of the fraction of QDs containing a hole in the $1 S_{\mathrm{h}}$ valence band state and the fraction of QDs containing an electron in the $1 S_{\mathrm{e}}$ conduction band state and is therefore a measure of the bleach at the band edge. The parameter $\delta E(t)$ is the time-dependent shift in energy of the $1 S_{\mathrm{h}}-1 \mathrm{~S}_{\mathrm{e}}$ transition of a photoexcited QD. As was discussed in literature this shift in energy and the function $f(t)$ can be determined independently. ${ }^{29,36}$ The shift $\delta E(t)$ is largest for hot excitons that are present on short time scales and decreases to a constant when all excitons have cooled down to the band edge. The factor $3 / 4$ brings into account the 8 -fold degeneracy of the $1 S_{h}$ and $1 S_{e}$ states and the fact that the bleach results from both reduced absorption and stimulated emission. ${ }^{13,29,40,42}$ Equation 1 can be rearranged to obtain the TA signal around the band edge bleach, which is given by

$$
\begin{gathered}
\Delta A=A^{*}-A=-f(t) C \mathrm{e}^{-\left(E-E_{0}\right)^{2} / 2 \sigma^{2}} \\
+\frac{3}{4} f(t) C \mathrm{e}^{-\left(E-\left(E_{0}-\delta E(t)\right)\right)^{2} / 2 \sigma^{2}}
\end{gathered}
$$

Fitting eq 2 to the TA spectrum at each pump-probe delay yields the time-dependent function $f(t)$. Note that when CM occurs the presence of two or more excitons in some QDs is included in the value of $f(t)$.

Fits of eq 2 to the TA spectrum of Figure 1 around the $1 S_{h}-$ $1 S_{\mathrm{e}}$ transition for short and long pump-probe delay are shown in Figure 2a. The fits reproduce the bleach of the $1 S_{h}-1 S_{e}$ transition and are truncated at the high-energy side to avoid effects of higher energy transitions that are not included in eq 2. The red curve in Figure $2 \mathrm{~b}$ shows the time-dependent function $f(t)$ obtained from the fit, as well as exponential fits to describe $f(t)$ as discussed below. The uncertainty in the fit of eq 2 at a particular pump-probe delay gives rise to noise in the function $f(t)$ of approximately $10 \%$.

In previous studies a single exponential function (of the form $1-\mathrm{e}^{-k t}$, which is characteristic for a first order decay process) has been used to describe cooling of hot charge carriers to the band edge states. ${ }^{12,29-31}$ As can be seen in Figure $2 b$, a fit of such a single exponential function does not reproduce $f(t)$. This is not surprising, because we previously found that holes cool much faster than electrons and therefore a sum of at least two exponential functions would be needed. ${ }^{32}$ However, we show in Figure $2 \mathrm{~b}$ that a double exponential function does not reproduce $f(t)$ either. Rather, the "S-like" shape of $f(t)$ indicates that cooling of electrons and holes to the band edge states takes place in several sequential steps. Thus, a model to describe $f(t)$ must include successive cooling steps of charge carriers from higher energy levels to the band edge states. ${ }^{39}$ In the next paragraph we discuss the analysis of our experimental data on the basis of such a kinetic model for electron and hole cooling in PbSe QDs.

Determination of Electron and Hole Cooling Times between Successive Energy Levels. We can describe charge carrier cooling as a sequence of steps between successive
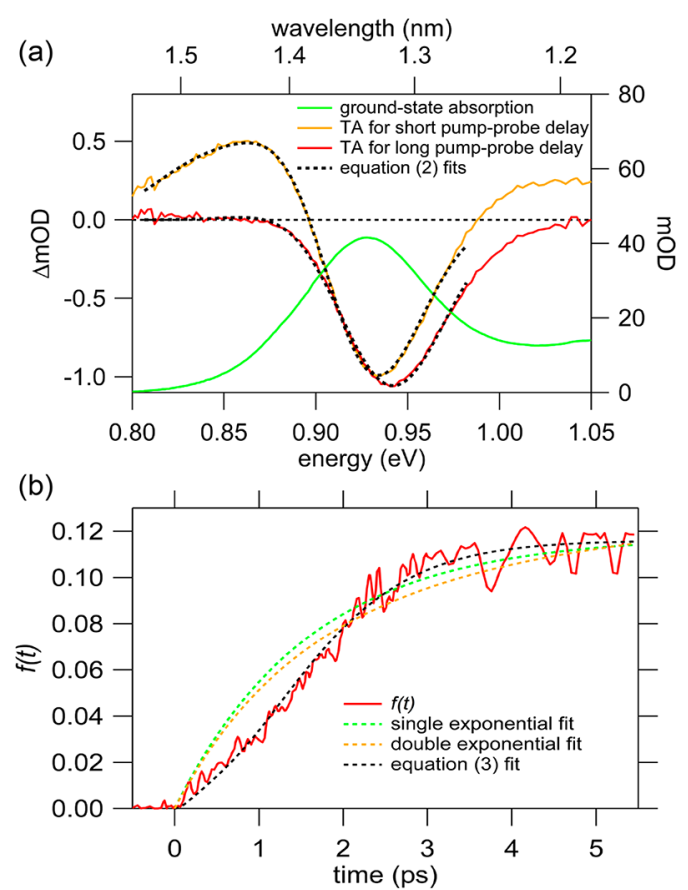

Figure 2. (a) TA spectrum around the band edge bleach for $3.9 \mathrm{~nm}$ QDs excited by a pump laser pulse with photon energy of $3.35 \mathrm{eV}$ $(370 \mathrm{~nm})$ for short and long pump-probe delay, together with fits of eq 2. (b) The function $f(t)$ obtained from fitting eq 2 to the experimental data, as well as exponential fits to describe $f(t)$ as discussed in the text.

electronic energy levels as schematically shown in Figure 3a. The time-dependent population of the different electronic states can be described by kinetic equations for relaxation of the electron and the hole, given by

$$
\begin{aligned}
\frac{\mathrm{d} N_{i, \mathrm{e}}(t)}{\mathrm{d} t} & =\frac{1}{\tau_{i+1, \mathrm{e}}} N_{i+1, \mathrm{e}}(t)-\frac{1}{\tau_{i, \mathrm{e}}} N_{i, \mathrm{e}}(t) \\
\frac{\mathrm{d} N_{i, \mathrm{~h}}(t)}{\mathrm{d} t} & =\frac{1}{\tau_{i+1, \mathrm{~h}}} N_{i+1, \mathrm{~h}}(t)-\frac{1}{\tau_{i, \mathrm{~h}}} N_{i, \mathrm{~h}}(t)
\end{aligned}
$$

with $N_{i, \mathrm{e}}$ the population of electrons in level $\mathrm{e}_{i}$ and $N_{i, \mathrm{~h}}$ the population of holes in level $\mathrm{h}_{i}$. The function $f(t)$ as shown in Figure $2 \mathrm{~b}$ can then be described as

$$
f(t)=A_{\mathrm{e}} N_{0, \mathrm{e}}(t)+A_{\mathrm{h}} N_{0, \mathrm{~h}}(t)
$$

with $A_{\mathrm{e}}\left(A_{\mathrm{h}}\right)$ the relative contribution of electrons (holes) to $f(t)$ and therefore to the bleach at the band edge.

We obtain the time constants $\left(\tau_{i, \mathrm{e}}, \tau_{i, \mathrm{~h}}\right)$ for the different cooling steps by fitting eq 3 to experimental data for $f(t)$, such as those shown in Figure 2 and Figure 3, while increasing the photoexcitation energy in small steps. For multiple photoexcitation energies we find that the time constants for specific transitions remain unchanged. Whenever the set of coupled rate equations requires the time constants to change in order to describe $f(t)$ accurately, we fix the time constants obtained thus far and add a next hole and electron level. We stop adding more discrete energy levels in the case where the time constant remains within the experimental time resolution of $\sim 0.15$ ps or increases continuously with photoexcitation energy. From that energy we consider the electronic structure of the $\mathrm{PbSe}$ QDs to resemble a quasi-continuum. In Figure 3 panels b-e we show 
(a)
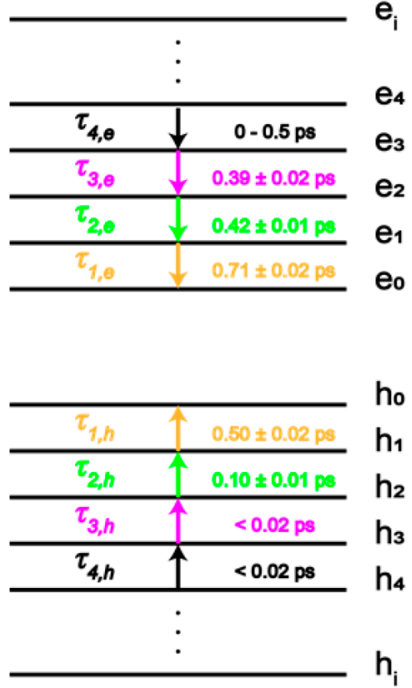

(b)

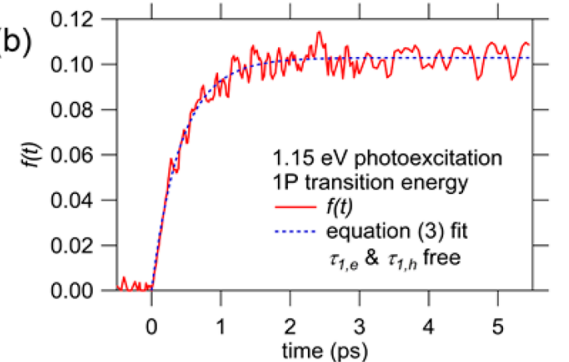

(d)

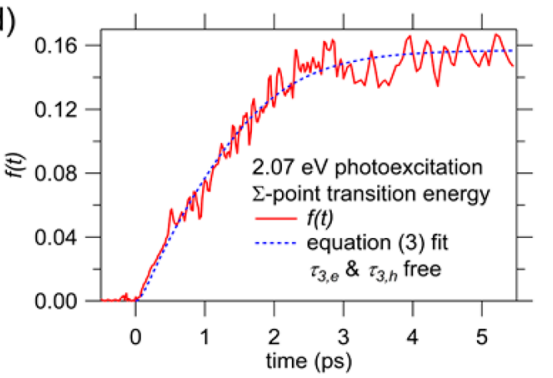

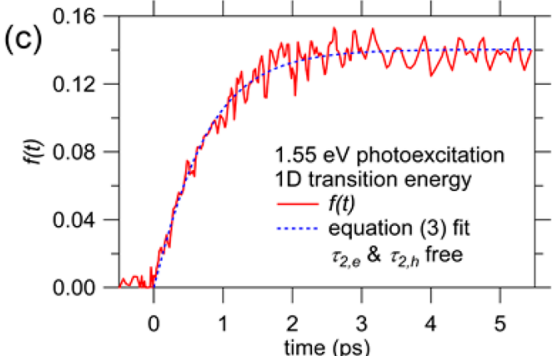

(e)

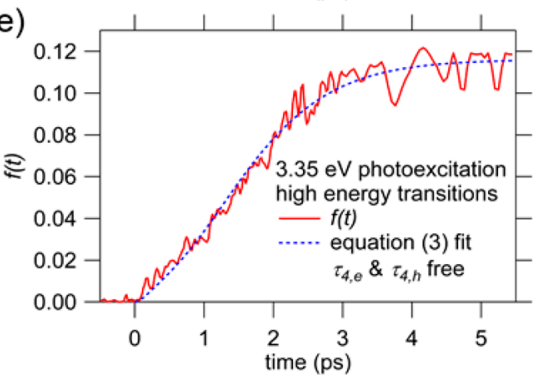

Figure 3. (a) Schematic model of the PbSe QD electronic structure with cooling time constants for transitions between successive energy levels. (b-e) Fits of eq 3 to measured data for $f(t)$ for photoexcitation energies and transitions as indicated, which are just above the threshold at which a new level and cooling time had to be included in eq 3 .

$f(t)$ with fits for photoexcitation energies just above successive thresholds at which a new energy level had to be included in eq 3. These photoexcitation energies coincide with the transitions in the ground-state absorption spectrum in Figure 1.

When photoexciting at $1.15 \mathrm{eV}$ (resonant with the $1 \mathrm{P}_{\mathrm{h}}-1 \mathrm{P}_{\mathrm{e}}$ transition) we can reproduce the measured $f(t)$ by including one cooling step for the electron (from level $e_{1}$ to $e_{0}$ ) and one for the hole (from level $h_{1}$ to $h_{0}$ ) in eq 3 , see Figure $3 b$. This results in a double exponential function of which one exponential describes the first electron cooling step (with cooling time $\tau_{1, \mathrm{e}}$ from $\mathrm{e}_{1}$ to $\mathrm{e}_{0}$ ) and the other the analogous first hole cooling step. We distinguish between electron and hole, as described in the next paragraph. The measured $f(t)$ in Figure $3 c$ for photoexcitation at $1.55 \mathrm{eV}$ can only be reproduced by adding a second cooling step for the electron and hole, while the first cooling times $\tau_{1, \mathrm{e}}$ and $\tau_{1, \mathrm{~h}}$ are kept fixed to the value obtained for lower photoexcitation energies. We continue with this procedure until we no longer find discrete cooling steps between energy levels. For electrons we find that $\tau_{4, \mathrm{e}}$ increases continuously with photoexcitation energy. Instead of defining a new energy level for each photoexcitation energy, we consider the electronic structure above the fourth electronic energy level $\left(\mathrm{e}_{4}\right)$ to approach a quasi-continuum. For holes we find that $\tau_{3, \mathrm{~h}}$ and higher are much smaller than our time resolution, and we therefore consider the electronic structure for holes to approach a quasi-continuum above the third electronic energy level $\left(h_{3}\right)$. The cooling times determined from the procedure described above are given between the corresponding energy levels in Figure 3a. We note that in our data, we have a rise time of $f(t)$ due to the pulse width of our laser. In Figure S2 of the Supporting Information we show $f(t)$ for band edge excitation where cooling is absent and determine an experimental time resolution of 0.15 ps from the rise time. The cooling time constants we list in Figure 3 are convolved with this time resolution.

We attributed cooling times to electrons or holes by making use of the high energy $\mathrm{L}_{4-6}$ and $\mathrm{L}_{5-7}$ transitions studied in our previous work that are visible as bleach features at $400-500 \mathrm{~nm}$ in Figure $1 .^{32}$ These are transitions from the second valence band to the first conduction band $\left(\mathrm{L}_{4-6}, 2.7 \mathrm{eV}\right)$ and from the first valence band to the second conduction band $\left(\mathrm{L}_{5-7}, 2.5\right.$ $\mathrm{eV})$. These transitions are selectively sensitive either to the $1 \mathrm{~S}_{\mathrm{e}}$ electron $\left(\mathrm{L}_{4-6}\right)$ or the $1 \mathrm{~S}_{\mathrm{h}}$ hole $\left(\mathrm{L}_{5-7}\right)$ at the band edge, when photoexciting with energy lower than these transition energies. In Figure $4 \mathrm{a}$ we show $f(t)$ for $1.77 \mathrm{eV}(700 \mathrm{~nm})$ photoexcitation together with the fit of eq 3 to $f(t)$. For the same photoexcitation energy we show a separate electron and hole bleach transient as determined from the $\mathrm{L}_{4-6}$ and $\mathrm{L}_{5-7}$ transitions in Figure $4 \mathrm{~b}$. The black curves in Figure $4 \mathrm{~b}$ are
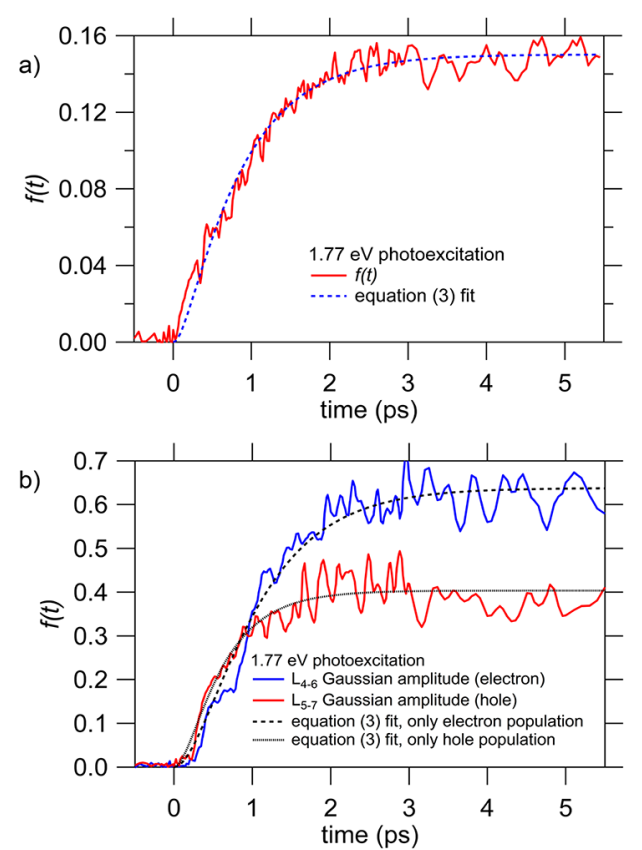

Figure 4. (a) Fit of eq 3 to $f(t)$ for $1.77 \mathrm{eV}$ photoexcitation of 3.9 $\mathrm{nm} \mathrm{PbSe}$ QDs. (b) Separate electron and hole bleach as determined from the high energy $L_{4-6}(2.7 \mathrm{eV})$ and $\mathrm{L}_{5-7}(2.5 \mathrm{eV})$ transitions described by the electron $\left(\mathrm{L}_{4-6}\right)$ and hole $\left(\mathrm{L}_{5-7}\right)$ contributions in eq $3 c$. 


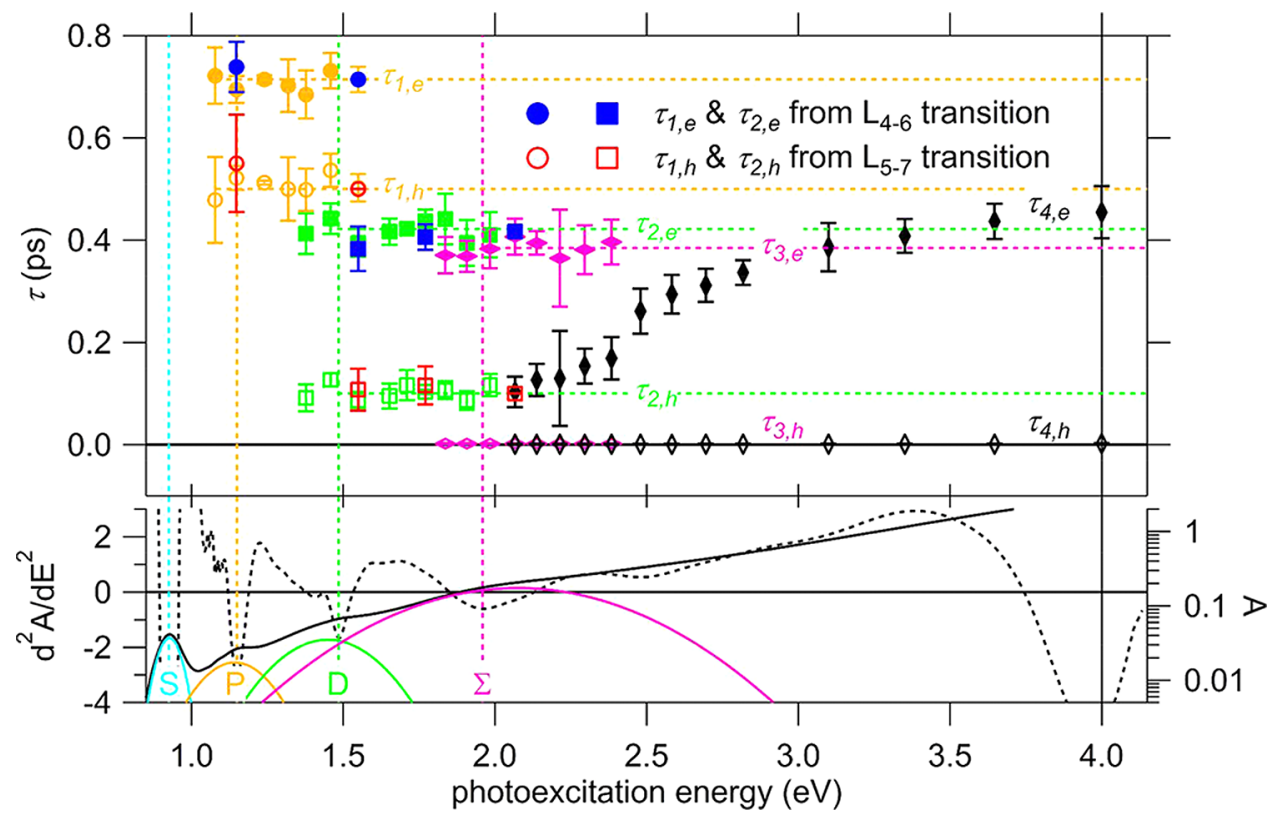

Figure 5. Broadband cooling spectrum for $3.9 \mathrm{~nm}$ PbSe QDs for electrons (filled symbols) and holes (open symbols). The optical absorption spectrum with its second derivative is included to obtain the position of the optical transitions, as indicated by the vertical dashed lines. Data from the $\mathrm{L}_{4-6}$ and $\mathrm{L}_{5-7}$ transitions allow us to attribute cooling times to electrons or holes.

the separate contributions of eq $3 \mathrm{c}$ due to electrons $\left(A_{\mathrm{e}} N_{0, \mathrm{e}}(t)\right)$ or holes $\left(A_{\mathrm{h}} N_{0, h}(t)\right)$ as obtained from the fit of eq 3 to $f(t)$ in Figure $4 \mathrm{a}$. As can be observed, the $\mathrm{L}_{4-6}$ bleach due to $1 \mathrm{~S}_{\mathrm{e}}$ electrons is very well described by one set of cooling times. Likewise the $\mathrm{L}_{5-7}$ bleach due to $1 \mathrm{~S}_{\mathrm{h}}$ holes is properly described by the other set of cooling times. Therefore, we can safely ascribe each set of cooling times to the corresponding charge carrier. We observe that electrons cool slower than holes in agreement with our previous work. ${ }^{32}$

Equation $3 \mathrm{c}$ also yields the relative contribution $A_{\mathrm{e}} / A_{\mathrm{h}}$ to $f(t)$ of electrons and holes. We show $A_{\mathrm{e}} / A_{\mathrm{h}}$ as a function of photoexcitation energy in Figure $\mathrm{S} 3$ of the Supporting Information. We find that $A_{\mathrm{e}} / A_{\mathrm{h}}$ is approximately 1.2 for all photoexcitation energies up to $2.1 \mathrm{eV}$, comparable to the relative contribution of electrons and holes to the band edge bleach as determined in our previous work using an electron scavenging molecule. ${ }^{32}$ For photoexcitation energies exceeding $2.1 \mathrm{eV}$ however, $A_{\mathrm{e}} / A_{\mathrm{h}}$ increases to values up to 3 . We believe that our model shown in Figure $3 \mathrm{a}$ is no longer valid at higher photoexcitation energy due to additional relaxation pathways for electrons and holes such as CM and the simultaneous excitation of various electron and hole states at different points in the band structure (e.g., with $\Sigma$ or W character). We therefore no longer assign electrons and holes to the two contributions, but rather realize that we have a slow and a fast cooling component that consists of both electrons and holes. We point out that this happens above $2.1 \mathrm{eV}$ photoexcitation energy where our results suggest that the electronic structures of both electrons and holes approach a quasi-continuum. The constant cooling times found between energy levels near the band edge remain valid.

The procedure described above yields the time constants for electron and hole cooling steps between successive discrete energy levels, as well as the cooling times from energies in the quasi-continuum to the highest discrete energy level that is distinguished. Taken together this constitutes a broadband cooling spectrum as shown in the upper panel of Figure 5. We also indicate the position of the optical transitions, as determined from the absorption spectrum in the lower panel of Figure 5 and include electron and hole cooling times as obtained from the $\mathrm{L}_{4-6}$ and $\mathrm{L}_{5-7}$ transitions, which agree with the data resulting from fitting eq 3 to $f(t)$.

Figure 5 shows that the $1 \mathrm{P}-1 \mathrm{~S}$ cooling times for electrons and holes are discrete and fully account for cooling up to $h \nu \approx$ $1.4 \mathrm{eV}$. At that point discrete $1 \mathrm{D}-1 \mathrm{P}$ cooling channels become available, followed by $\Sigma-1 \mathrm{D}$ channels at $h \nu \approx 1.9 \mathrm{eV}$. From that point on the cooling times increase continuously as the density-of-states (DOS) forms a quasi-continuum. Figure 5 further shows that each individual hole cooling step is faster than the corresponding step for an electron. This agrees with our earlier finding that the total cooling time from high energy down to the band edge is shorter for a hole than for an electron. ${ }^{32}$ A second finding is the decrease of both the electron and hole cooling time constants on going from the lowest energy transition to higher transitions. The electron cooling time constants for transitions between discrete levels decrease slightly with energy but remain in the same order of magnitude of $\sim 0.5$ ps. The hole cooling time constant for the first transition is slightly smaller than that for the electron. Interestingly, the hole cooling time constants for the next transitions are significantly shorter than the corresponding electron cooling time constants and have values within the $\sim 0.15$ ps time resolution of our experiment.

Factors Governing Electron and Hole Cooling Times. According to calculations in the literature, emission of LO phonons mediates charge carrier cooling in $\mathrm{PbSe} \mathrm{QDs}$ at high excess energy. ${ }^{11,16,43}$ The energy of LO phonons in PbSe is $\sim 17 \mathrm{meV}$ and the typical time of one LO phonon emission event in bulk PbSe is $\sim 25$ fs. ${ }^{43}$ For large charge carrier excess energy, the quasi-continuum of electronic states will contain levels with spacing (nearly) equal to an LO phonon energy and charge cooling via LO phonon emission can be efficient. However, for the lowest cooling steps near the band gap the energy difference between the discrete electronic states $(>100$ 


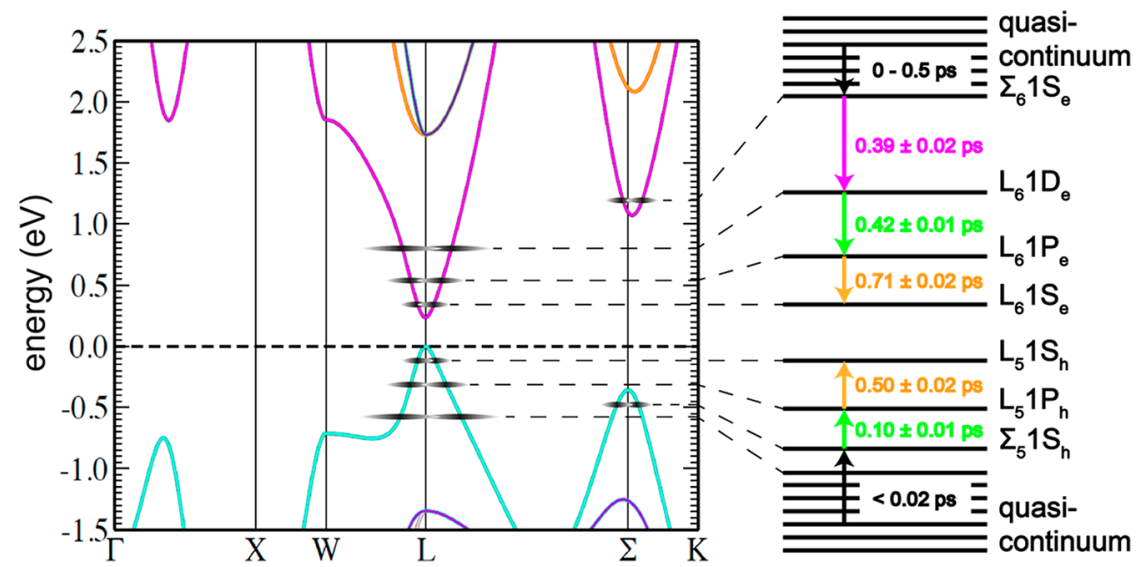

Figure 6. PbSe bulk band structure (left) and schematic PbSe QD electronic structure (right) including our experimental cooling time constants.

$\mathrm{meV}$ ) is much larger than the LO phonon energy. To overcome these large electronic energy differences, each cooling step would require simultaneous emission of multiple LO phonons. Theoretical calculations show that this is very unlikely and would lead to cooling times in the order of nanoseconds, which is orders of magnitude longer than our findings. ${ }^{44}$

In addition to LO phonon emission, charge carrier cooling can be facilitated by energy transfer to vibrational modes of the oleate ligands. In agreement with the latter it was found for CdSe QDs that ligands have a large influence on charge carrier cooling. ${ }^{22,23}$ In Figure S4 of the Supporting Information we show an FTIR spectrum of our PbSe QDs with oleate ligands, revealing oleate vibrational modes in the energy range of 100$200 \mathrm{meV}$ and near $350 \mathrm{meV}$ (corresponding to the carbonyl stretch vibration and $\mathrm{CH}$ stretch vibrations in the aliphatic chain, respectively). We also show electronic transition energies as obtained from the optical absorption spectrum that overlap with the ligand vibration energies. Hence, energy transfer to ligand vibrational modes can facilitate charge carrier cooling near the band edge. Beside ligand vibrational modes, the surface of QDs also enables other strong phonon interactions that could assist charge carrier cooling.

The above considerations do not explain why holes cool much faster than electrons. To gain more insight into carrier relaxation by $\mathrm{LO}$ phonon emission we calculate the $\mathrm{PbSe}$ bulk band structure and $\mathrm{QD}$ electronic structure using a $k \cdot \boldsymbol{p}$ Hamiltonian with states around both the L-point and the $\Sigma$ point in the first Brillouin Zone of the rock-salt lattice. ${ }^{45-48} \mathrm{We}$ parametrize this Hamiltonian using $a b$ initio DFT to solve the Kohn-Sham equation for bulk PbSe in the CASTEP plane waves based parallel DFT code. ${ }^{49,50}$ We use the TB-mBJ exchange potential with treatment of spin-orbit interactions at the scalar (or vector, see Supporting Information) relativistic level (see Methods). ${ }^{51}$ We show the calculated bulk band structure including a schematic QD electronic structure to incorporate our experimental cooling time constants in Figure 6. In Figure S5 of the Supporting Information we include a comparison of the calculated QD electronic structure with treatment of spin-orbit interactions both at the scalar and vector relativistic level showing the same qualitative behavior.

From Figure 6 and Figure S5 of the Supporting Information we observe that the first electronic energy levels in the QDs in the conduction band arise predominantly from the bulk Lpoint, followed by energy levels that also have $\Sigma$-point character. This gives rise to a quasi-continuum. In the valence band the $\Sigma$-point is mixed into the QD electronic structure at much lower energy, resulting in a much lower onset of the quasi-continuum. As first suggested by Zunger et al., ${ }^{52}$ this is caused by the smaller energy difference between the $\mathrm{L}$ and $\Sigma$ extrema in the valence band than in the conduction band, which is also evident in Figure 6. The result is a higher DOS in the valence band than in the conduction band, in line with previous results from Zunger ${ }^{52}$ and Liljeroth, ${ }^{53}$ resulting in faster hole cooling rates. These observations agree very well with the experimental cooling time constants and corresponding optical transitions in Figure 5.

To describe charge carrier cooling in the quasi-continuum, the energy loss rate $\gamma$ of electrons and holes at high excess energy above the first $\Sigma$-point level can be obtained from the experiments using

$$
\gamma=\frac{\mathrm{d} E}{\mathrm{~d} t}=\left[\frac{\mathrm{d} \tau}{\mathrm{d}(h v)}\right]^{-1}
$$

where $\tau$ is the cooling time and $h \nu$ is the photoexcitation energy. If it is assumed that the photon energy in excess of the band gap is equally distributed over the electron and the hole (admittedly a strong simplification considering the discussion above), the data in Figure 5 yield an energy loss rate for the electron of $1 \mathrm{eV} / \mathrm{ps}$ at low energy, increasing to $5 \mathrm{eV} / \mathrm{ps}$ at high energy. This seems reasonable considering previous calculations. ${ }^{1,16,43}$ The hole cooling time is faster than the $\sim 0.15$ ps experimental time resolution, leading to an energy loss rate of at least $8 \mathrm{eV} / \mathrm{ps}$ even near the bottom of the quasi-continuum. This very high energy loss rate for holes could be due to additional relaxation pathways such as CM not considered in eq 3. In addition, an asymmetric division of the energy between electron and hole due to the difference in DOS puts most energy in the electron, enhancing the apparent difference in energy loss rates. When photoexcitation occurs via the $\mathrm{L}_{4-6}$ or the $\mathrm{L}_{5-7}$ transition, ${ }^{32}$ all excess energy is transferred either to the hole or the electron and a very asymmetric division of energy is achieved. These effects occur above the $\Sigma$-point transition in the quasi-continuum and do not affect the cooling time constants we find between discrete energy levels near the band edge.

If charge carrier cooling in the quasi-continuum occurs solely due to emission of LO phonons with an energy of $\sim 17$ $\mathrm{meV},{ }^{11,16,43}$ the energy loss rate for electrons of $1-5 \mathrm{eV} / \mathrm{ps}$ leads to a net LO phonon emission time of 3.4-17 fs. Likewise 
the energy loss rate for holes of at least $8 \mathrm{eV} / \mathrm{ps}$ leads to a net LO phonon emission time $<2.1$ fs. Using the calculated electronic states from Figure S5, we can describe carrier relaxation in $\mathrm{PbSe} \mathrm{QDs}$ through emission of $\mathrm{LO}$ phonons with the Wigner-Weisskopf description in the strong coupling regime (see Methods). ${ }^{54}$ We assume LO phonon decay through the Ridley LO $\rightarrow \mathrm{TO}+\mathrm{TA}$ channel due to the large anharmonicity of LO phonons in the rock-salt structure (see Methods). ${ }^{55-57}$ We find the shortest LO phonon emission time for resonance of the transition energy with the LO phonon energy of $17 \mathrm{meV}$. This emission time is $3.6 \mathrm{fs}$ and agrees well with our experimental energy loss rates mentioned above.

\section{CONCLUSIONS}

We identify five discrete cooling steps (three for electrons and two for holes) before the onset of a continuously increasing cooling time in PbSe QDs. The cooling time constants for both electrons and holes between discrete electronic states close to the band edge are of the order of 0.5 ps and require energy transfer to surface ligand vibrational modes or surface phonon modes. At high excess energy electrons and holes cool via emission of LO phonons due to the convergence of the electronic structure to a quasi-continuum. There the experimental energy loss rate is $1-5 \mathrm{eV} / \mathrm{ps}$ for electrons and at least $8 \mathrm{eV} / \mathrm{ps}$ for holes. These results agree well with theoretical carrier relaxation results. We find the start of the quasicontinuum for holes much closer to the band edge than for electrons and therefore confirm an asymmetric density-of-states for PbSe QDs. The results constitute the first broadband cooling spectrum for electrons and holes in colloidal nanocrystals.

\section{METHODS}

Synthesis of PbSe QDs. PbSe QDs with a diameter of $3.9 \mathrm{~nm}$ were synthesized using the method described by Steckel et al. ${ }^{33}$ In brief, $4.77 \mathrm{~g}$ of lead acetate trihydrate (99.999\%, Aldrich), $3.42 \mathrm{~g}$ of oleic acid (90\%, Aldrich) and $13.14 \mathrm{~g}$ of 1-octadecene (90\%, Aldrich) were placed into a flask and dried and reacted under vacuum for at least $2 \mathrm{~h}$ at $120^{\circ} \mathrm{C}$. A second mixture containing $1.12 \mathrm{~g}$ of selenium (99.999\%, Alfa Aesar), $0.13 \mathrm{~mL}$ of diphenylphospine (98\%, Aldrich), and $14.87 \mathrm{~mL}$ of trioctylphosphine (90\%, Fluka) was prepared. Subsequently, the lead mixture was heated to $180^{\circ} \mathrm{C}$ under nitrogen and the selenium mixture was injected. The reaction was allowed to proceed for $10 \mathrm{~s}$ at $150{ }^{\circ} \mathrm{C}$ after which the reaction was quenched using $15 \mathrm{~mL}$ of butanol (99.8 anhydrous, Aldrich). The crude synthesis mixtures were washed twice by precipitating with methanol, centrifugation, and redispersion of the sediment in toluene. This resulted in particles with a diameter of $3.9 \pm 0.3 \mathrm{~nm}$ as determined by TEM.

Hyperspectral TA Spectroscopy. We studied charge carrier cooling in PbSe QDs using broadband optical pump-probe spectroscopy. The QD samples were dispersed in toluene in a 2 $\mathrm{mm}$ stirred quartz cuvette at a typical optical density of 0.05 at the band gap. The QD dispersion was stirred during TA experiments to prevent photocharging.

Femtosecond laser pulses were generated in a $\mathrm{Yb}: \mathrm{KGW}$ oscillator (Light Conversion, Pharos SP) at $1028 \mathrm{~nm}$ and amplified. A small fraction of the $1028 \mathrm{~nm}$ fundamental beam was split off to generate the broadband probe spectrum in a sapphire $(500-1600 \mathrm{~nm})$ or $\mathrm{CaF}_{2}$ $(375-600 \mathrm{~nm})$ crystal. The probe pulse was delayed up to $2.5 \mathrm{~ns}$ using an automated delay stage. The majority of the $1028 \mathrm{~nm}$ fundamental beam was used as a pump pulse after nonlinear frequency mixing in an OPA and second harmonics module (Light Conversion, Orpheus) to achieve wavelengths of $310-1330 \mathrm{~nm}$. The pump and probe pulses overlap on the sample position under an angle of $\sim 8$ degrees, after which the pump pulse is dumped and the probe light is led to a detector suitable for the probe spectrum selected (Ultrafast Systems, Helios). We calculate the pump-induced change in absorption according to

$$
\Delta A=A_{\text {on }}-A_{\text {off }}=\log \left(\frac{I_{0}}{I_{\text {on }}}\right)-\log \left(\frac{I_{0}}{I_{\text {off }}}\right)=\log \left(\frac{I_{\text {off }}}{I_{\text {on }}}\right)
$$

with $I_{0}$ the intensity of the probe beam incident on the sample and $I_{\text {on }}$ and $I_{\text {off }}$ the intensity of the probe beam transmitted through the sample with the pump beam either on or off. All shown data is corrected for dispersion by fitting a polynomial function to the solvent response. The time resolution of the TA measurements is determined by the rise time of the TA signal at band edge excitation as shown in Figure S2 of the Supporting Information and is 0.15 ps.

In all experiments the laser pump fluence was taken sufficiently low to prevent multiple photons being absorbed in a single QD. Assuming Poissonian statistics for photoexcitation, the probability $P_{N}$ for a QD to absorb $N$ photons is given by

$$
P_{N}=\frac{\mathrm{e}^{\left\langle-N_{\mathrm{abs}}\right\rangle}\left\langle N_{\mathrm{abs}}\right\rangle^{N}}{N !}
$$

with the average number of photons absorbed per QD equal to $\left\langle N_{\mathrm{abs}}\right\rangle$ $=J \sigma$ where $J$ is the laser pump fluence and $\sigma$ is the photon absorption cross section. During the measurements we take $J$ low enough, so that $P_{N \geq 2}$ is negligible, that is, $\left\langle N_{\text {abs }}\right\rangle<0.15$, and $P_{N \geq 2}=1-P_{0}-P_{1}=0.01$.

Electronic Structure Calculations. We calculate the electronic structure of $\mathrm{PbSe}$ QDs using a $\boldsymbol{k} \cdot \boldsymbol{p}$ Hamiltonian with states around the L-point in the first Brillouin zone of the rock-salt lattice, ${ }^{45-48}$ augmented with states around the $\Sigma$-point. This Hamiltonian is parametrized using $a b$ initio DFT (without fitting the band structure) to solve the Kohn-Sham equation in the CASTEP plane waves based parallel DFT code. ${ }^{49,50}$ Many electron effects are approximated using the Tran-Blaha (TB-mBJ) exchange correlation functional as implemented in CASTEP. ${ }^{47,51}$ Spin-orbit effects are taken into account at the scalar (vector) relativistic level. For parametrization of the $k \cdot p$ Hamiltonian we use the room temperature value of the effective masses at the L-point, while at the $\Sigma$-point we use those obtained from DFT. ${ }^{58}$ This leads to good agreement between the experimental $(\sim 110 \mathrm{meV}$ if assumed equal for electron and hole $)$ and theoretical $(147 \mathrm{meV}) 1 \mathrm{P}_{\mathrm{e}}-1 \mathrm{~S}_{\mathrm{e}}$ transition. We find that the energetic difference between the top of the valence band at the $\Sigma$-point and the top of the valence band at the L-point is much smaller than the energetic difference between the corresponding band extrema in the conduction band. This results in a higher density-of-states in the valence band. Energy gaps and effective masses are given in the Supporting Information.

Electron-Phonon Scattering Calculations. To describe charge carrier cooling by the emission of LO phonons, we follow the Wigner-Weisskopf description in the strong coupling regime. This allows us to couple electrons to the LO phonon when the energy between electronic energy levels is not resonant with the LO phonon energy. We can then calculate the phonon emission rate by ${ }^{54}$

$$
\begin{aligned}
\frac{1}{\tau_{\mathrm{if}}^{\mathrm{ph}}} & =W_{\mathrm{ph}}-\frac{\sqrt{2}}{\hbar}\left(\left[\left(\frac{g_{\mathrm{if}}^{2}}{\hbar^{2}}+\frac{\Delta_{\mathrm{if}}^{2}-\left(\hbar W_{\mathrm{ph}}\right)^{2}}{4}\right)^{2}+\frac{\left(\hbar W_{\mathrm{ph}} \Delta_{\mathrm{if}}\right)^{2}}{2}\right]^{1 / 2}\right. \\
- & \left.-\left(\frac{g_{\mathrm{if}}^{2}}{\hbar^{2}}+\frac{\Delta_{\mathrm{if}}^{2}-\left(\hbar W_{\mathrm{ph}}\right)^{2}}{4}\right)\right)^{1 / 2}
\end{aligned}
$$

Here $\Delta_{\mathrm{if}}=E_{\mathrm{i}}-E_{\mathrm{f}} \pm \hbar \omega_{\mathrm{LO}}$ is the detuning of the LO phonon energy $\hbar \omega_{\mathrm{LO}}$ from the difference in energy between the initial and final electronic energy levels $E_{\mathrm{i}}$ and $E_{\mathrm{f}}, W_{\mathrm{ph}}$ is the phenomenological rate of LO phonon decay into two less energetic phonons due to anharmonicity and $g_{\text {if }}$ is the coupling strength of an electron to the LO phonon. Assuming the Fröhlich interaction for coupling between electrons and LO phonons $g_{\text {if }}$ is given by 


$$
g_{\text {if }}^{2}=\sum_{\mathbf{q}}\left(n_{\mathrm{LO}}+\frac{1}{2} \pm \frac{1}{2}\right)|\alpha(\mathbf{q})|^{2}\left|F_{i f}(\mathbf{q})\right|^{2}
$$

where $n_{\mathrm{LO}}$ is the phonon occupation number, $F_{\mathrm{if}}(\boldsymbol{q})=\left\langle i e^{i q r} \mid f\right\rangle$ is the electron-phonon coupling matrix element, and $q$ is the phonon momentum vector. We take care of momentum conservation through $\mathbf{q} \rightarrow \mathbf{q}+|\overrightarrow{L \Sigma}|$ with $|\overrightarrow{L \Sigma}|=(3 / 2 \sqrt{2})\left(\pi / a_{0}\right) \sim 0.544 \AA^{-1}$ while calculating $\tau_{\text {if }}^{\text {ph }}$ according to eqs 7 and 8 . We assume that the Ridley $\mathrm{LO} \rightarrow \mathrm{TO}+\mathrm{TA}$ channel is the most dominant LO phonon decay channel, significantly faster than any other channel such as Klemens or Vallee-Bogani, ${ }^{55-57}$ due to the large Grüneisen parameter of the TO mode in PbSe, $\gamma(\mathrm{TO})=15 .{ }^{59}$ For this channel we estimate $W_{\mathrm{ph}} \sim 277$ $\mathrm{ps}^{-1}$ at room temperature.

\section{ASSOCIATED CONTENT}

\section{S Supporting Information}

The Supporting Information is available free of charge on the ACS Publications website at DOI: 10.1021/acsnano.7b02506.

Detailed comparison between integration of TA spectra and the fitting method of eq 2 that we apply to show the identical time evolution of the band edge bleach found from these methods; determination of the rise time of $f(t)$ for band edge excitation; information on the relative contribution of the electron and hole components in our fit model as a function of excitation energy; FTIR spectrum showing the energy of vibrational modes in the QD ligands that overlap with transitions between discrete electronic energy levels of the QD; calculations of the $\mathrm{PbSe} \mathrm{QD}$ electronic structure taking spin-orbit interactions into account at the scalar and vector relativistic levels (PDF)

\section{AUTHOR INFORMATION}

\section{Corresponding Authors}

*E-mail: A.J.Houtepen@tudelft.nl.

*E-mail: L.D.A.Siebbeles@tudelft.nl.

\section{ORCID}

Laurens D. A. Siebbeles: 0000-0002-4812-7495

\section{Author Contributions}

The experiments and fits were performed by F.C.M.S. Calculations were done by S.T. A.J.H. and L.D.A.S. managed the project.

\section{Notes}

The authors declare no competing financial interest.

\section{ACKNOWLEDGMENTS}

F.C.M.S. and L.D.A.S. were supported by the Dutch Foundation for Fundamental Research on Matter (FOM) with the project "Hot Electrons in Cool Nanocrystals". S.T. would like to thank the UK's EPSRC funding body for support through the project "Enhanced multiple exciton generation in colloidal quantum dots" (EP/K008587/1). S.T. would like to acknowledge the contribution of EU COST Action MP1406. The authors would like to thank P. Liljeroth and D. Vanmaekelbergh for discussions.

\section{REFERENCES}

(1) Talapin, D. V.; Lee, J. S.; Kovalenko, M. V.; Shevchenko, E. V. Prospects of Colloidal Nanocrystals for Electronic and Optoelectronic Application. Chem. Rev. 2010, 110, 389-458.
(2) Park, J.; Joo, J.; Kwon, S. G.; Jang, Y.; Hyeon, T. Synthesis of Monodisperse Spherical Nanocrystals. Angew. Chem., Int. Ed. 2007, 46, $4630-4660$.

(3) Smith, C.; Binks, D. Multiple Exciton Generation in Colloidal Nanocrystals. Nanomaterials 2014, 4, 19-45.

(4) Beard, M. C.; Luther, J. M.; Semonin, O. E.; Nozik, A. J. Third Generation Photovoltaics Based on Multiple Exciton Generation in Quantum Confined Semiconductors. Acc. Chem. Res. 2013, 46, 12521260.

(5) Padilha, L. A.; Stewart, J. T.; Sandberg, R. L.; Bae, W. K.; Koh, W.-K.; Pietryga, J. M.; Klimov, V. I. Carrier Multiplication in Semiconductor Nanocrystals: Influence of Size, Shape and Composition. Acc. Chem. Res. 2013, 46, 1261-1269.

(6) ten Cate, S.; Sandeep, C. S. S.; Liu, Y.; Law, M.; Kinge, S.; Houtepen, A. J.; Schins, J. M.; Siebbeles, L. D. A. Generating Free Charges by Carrier Multiplication in Quantum Dots for Highly Efficient Photovoltaics. Acc. Chem. Res. 2015, 48, 174-181.

(7) Stewart, J. T.; Padilha, L. A.; Bae, W. K.; Koh, W.-K.; Pietryga, J. M.; Klimov, V. I. Carrier Multiplication in Quantum Dots within the Framework of Two Competing Energy Relaxation Mechanisms. J. Phys. Chem. Lett. 2013, 4, 2061-2068.

(8) Stewart, J. T.; Padilha, L. A.; Qazilbash, M. M.; Pietryga, J. M.; Midgett, A. G.; Luther, J. M.; Beard, M. C.; Nozik, A. J.; Klimov, V. I. Comparison of Carrier Multiplication Yields in $\mathrm{PbS}$ and $\mathrm{PbSe}$ Nanocrystals: The Role of Competing Energy-Loss Processes. Nano Lett. 2012, 12, 622-628.

(9) Allan, G.; Delerue, C. Role of Impact Ionization in Multiple Exciton Generation in PbSe Nanocrystals. Phys. Rev. B: Condens. Matter Mater. Phys. 2006, 73, 205423.

(10) Kambhampati, P. Hot Exciton Relaxation Dynamics in Semiconductor Quantum Dots: Radiationless Transitions on the Nanoscale. J. Phys. Chem. C 2011, 115, 22089-22109.

(11) An, J. M.; Califano, M.; Franceschetti, A.; Zunger, A. ExcitedState Relaxation in PbSe Quantum Dots. J. Chem. Phys. 2008, 128, 164720.

(12) Schaller, R. D.; Pietryga, J. M.; Goupalov, S. V.; Petruska, M. A.; Ivanov, S. A.; Klimov, V. I. Breaking the Phonon Bottleneck in Semiconductor Nanocrystals via Multiphonon Emission Induced by Intrinsic Nonadiabatic Interactions. Phys. Rev. Lett. 2005, 95, 196401.

(13) Wehrenberg, B. L.; Wang, C. J.; Guyot-Sionnest, P. Interband and Intraband Optical Studies of PbSe Colloidal Quantum Dots. J. Phys. Chem. B 2002, 106, 10634-10640.

(14) Nozik, A. J. Quantum Dot Solar Cells. Phys. E 2002, 14, 115120.

(15) Nozik, A. J. Multiple Exciton Generation in Semiconductor Quantum Dots. Chem. Phys. Lett. 2008, 457, 3-11.

(16) Kilina, S. V.; Kilin, D. S.; Prezhdo, O. V. Breaking the Phonon Bottleneck in $\mathrm{PbS}$ and $\mathrm{CdSe}$ Quantum Dots: Time-Domain Density Functional Theory of Charge Carrier Relaxation. ACS Nano 2009, 3, 93-99.

(17) Peterson, M. D.; Cass, L. C.; Harris, R. D.; Edme, K.; Sung, K.; Weiss, E. A. The Role of Ligands in Determining the Exciton Relaxation Dynamics in Semiconductor Quantum Dots. Annu. Rev. Phys. Chem. 2014, 65, 317-339.

(18) Lifshitz, E. Evidence in Support of Exciton to Ligand Vibrational Coupling in Colloidal Quantum Dots. J. Phys. Chem. Lett. 2015, 6, 4336-4347.

(19) Bozyigit, D.; Yazdani, N.; Yarema, M.; Yarema, O.; Lin, W. M. M.; Volk, S.; Vuttivorakulchai, K.; Luisier, M.; Juranyi, F.; Wood, V. Soft Surfaces of Nanomaterials Enable Strong Phonon Interactions. Nature 2016, 531, 618-622.

(20) Guyot-Sionnest, P.; Hines, M. A. Intraband Transitions in Semiconductor Nanocrystals. Appl. Phys. Lett. 1998, 72, 686-688.

(21) Guyot-Sionnest, P.; Shim, M.; Matranga, C.; Hines, M. Intraband Relaxation in CdSe Quantum Dots. Phys. Rev. B: Condens. Matter Mater. Phys. 1999, 60, 2181-2184.

(22) Guyot-Sionnest, P.; Wehrenberg, B.; Yu, D. Intraband Relaxation in CdSe Nanocrystals and the Strong Influence of the Surface Ligands. J. Chem. Phys. 2005, 123, 074709. 
(23) Pandey, A.; Guyot-Sionnest, P. Slow Electron Cooling in Colloidal Quantum Dots. Science 2008, 322, 929-32.

(24) Cho, B.; Peters, W. K.; Hill, R. J.; Courtney, T. L.; Jonas, D. M. Bulklike Hot Carrier Dynamics in Lead Sulfide Quantum Dots. Nano Lett. 2010, 10, 2498-2505.

(25) Miaja-Avila, L.; Tritsch, J. R.; Wolcott, A.; Chan, W. L.; Nelson, C. A.; Zhu, X. Y. Direct Mapping of Hot-Electron Relaxation and Multiplication Dynamics in PbSe Quantum Dots. Nano Lett. 2012, 12, 1588-1591.

(26) Yang, J.; Hyun, B.-R.; Basile, A. J.; Wise, F. W. Exciton Relaxation in PbSe Nanorods. ACS Nano 2012, 6, 8120-8127.

(27) Gdor, I.; Sachs, H.; Roitblat, A.; Strasfeld, D. B.; Bawendi, M. G.; Ruhman, S. Exploring Exciton Relaxation and Multiexciton Generation in PbSe Nanocrystals Using Hyperspectral Near-IR Probing. ACS Nano 2012, 6, 3269-3277.

(28) Gdor, I.; Yang, C.; Yanover, D.; Sachs, H.; Lifshitz, E.; Ruhman, $\mathrm{S}$. Novel Spectral Decay Dynamics of Hot Excitons in $\mathrm{PbSe}$ Nanocrystals: A Tunable Femtosecond Pump-Hyperspectral Probe Study. J. Phys. Chem. C 2013, 117, 26342-26350.

(29) Gao, Y.; Talgorn, E.; Aerts, M.; Trinh, M. T.; Schins, J. M.; Houtepen, A. J.; Siebbeles, L. D. A. Enhanced Hot-Carrier Cooling and Ultrafast Spectral Diffusion in Strongly Coupled PbSe QuantumDot Solids. Nano Lett. 2011, 11, 5471-5476.

(30) Nootz, G.; Padilha, L. A.; Levina, L.; Sukhovatkin, V.; Webster, S.; Brzozowski, L.; Sargent, E. H.; Hagan, D. J.; Van Stryland, E. W. Size Dependence of Carrier Dynamics and Carrier Multiplication in PbS Quantum Dots. Phys. Rev. B: Condens. Matter Mater. Phys. 2011, 83,155302 .

(31) Ellingson, R. J.; Blackburn, J. L.; Nedeljkovic, J.; Rumbles, G.; Jones, M.; Fu, H.; Nozik, A. J. Theoretical and Experimental Investigation of Electronic Structure and Relaxation of Colloidal Nanocrystaline Indium Phosphide Quantum Dots. Phys. Rev. B: Condens. Matter Mater. Phys. 2003, 67, 075308.

(32) Spoor, F. C. M.; Kunneman, L. T.; Evers, W. H.; Renaud, N.; Grozema, F. C.; Houtepen, A. J.; Siebbeles, L. D. A. Hole Cooling is Much Faster than Electron Cooling in PbSe Quantum Dots. ACS Nano 2016, 10, 695-703.

(33) Steckel, J. S.; Yen, B. K.; Oertel, D. C.; Bawendi, M. G. On the Mechanism of Lead Chalcogenide Nanocrystal Formation. J. Am. Chem. Soc. 2006, 128, 13032-13033.

(34) Kigel, A.; Brumer, M.; Maikov, G. I.; Sashchiuk, A.; Lifshitz, E. Thermally Activated Photoluminescence in Lead Selenide Colloidal Quantum Dots. Small 2009, 5, 1675-1681.

(35) Ellingson, R. J.; Beard, M. C.; Johnson, J. C.; Yu, P.; Micic, O. I.; Nozik, A. J.; Shabaev, A.; Efros, A. L. Highly Efficient Multiple Exciton Generation in Colloidal PbSe and $\mathrm{PbS}$ Quantum Dots. Nano Lett. 2005, 5, 865-871.

(36) Geiregat, P.; Houtepen, A. J.; Justo, Y.; Grozema, F. C.; Van Thourhout, D.; Hens, Z. Coulomb Shifts upon Exciton Addition to Photoexcited PbS Colloidal Quantum Dots. J. Phys. Chem. C 2014, 118, 22284-22290.

(37) Trinh, M. T.; Houtepen, A. J.; Schins, J. M.; Piris, J.; Siebbeles, L. D. A. Nature of the Second Optical Transition in PbSe Quantum Dots. Nano Lett. 2008, 8, 2112-2117.

(38) Trinh, M. T.; Sfeir, M. Y.; Choi, J. J.; Owen, J. S.; Zhu, X. A Hot Electron-Hole Pair Breaks the Symmetry of a Semiconductor Quantum Dot. Nano Lett. 2013, 13, 6091-6097.

(39) Aerts, M.; Spoor, F. C. M.; Grozema, F. C.; Houtepen, A. J.; Schins, J. M.; Siebbeles, L. D. A. Cooling and Auger Recombination of Charges in PbSe Nanorods: Crossover from Cubic to Bimolecular Decay. Nano Lett. 2013, 13, 4380-4386.

(40) Trinh, M. T.; Houtepen, A. J.; Schins, J. M.; Hanrath, T.; Piris, J.; Knulst, W.; Goossens, A. P. L. M.; Siebbeles, L. D. A. In Spite of Recent Doubts Carrier Multiplication Does Occur In PbSe Nanocrystals. Nano Lett. 2008, 8, 1713-1718.

(41) Gdor, I.; Shapiro, A.; Yang, C.; Yanover, D.; Lifshitz, E.; Ruhman, S. Three-Pulse Femtosecond Spectroscopy of PbSe Nanocrystals: $1 S$ Bleach Nonlinearity and Sub-Band-Edge Excited-State Absorption Assignment. ACS Nano 2015, 9, 2138-2147.
(42) Schaller, R. D.; Petruska, M. A.; Klimov, V. I. Tunable NearInfrared Optical Gain and Amplified Spontaneous Emission Using PbSe Nanocrystals. J. Phys. Chem. B 2003, 107, 13765-13768.

(43) Geiregat, P.; Delerue, C.; Justo, Y.; Aerts, M.; Spoor, F. C. M.; Van Thourhout, D.; Siebbeles, L. D. A.; Allan, G.; Houtepen, A. J.; Hens, Z. A Phonon Scattering Bottleneck for Carrier Cooling in Lead Chalcogenide Nanocrystals. ACS Nano 2015, 9, 778-788.

(44) Dmitriev, I. A.; Suris, R. A. Anharmonicity-Assisted Multiphonon Transitions Between Distant Levels in Semiconductor Quantum Dots. Phys. Rev. B: Condens. Matter Mater. Phys. 2014, 90, 155431.

(45) Dimmock, J. O.; Wright, G. B. Band Edge Structure of PbS, PbSe and PbTe. Phys. Rev. 1964, 135, 821-830.

(46) Kang, I.; Wise, F. W. Electronic Structure and Optical Properties of PbS and PbSe Quantum Dots. J. Opt. Soc. Am. B 1997, 14, 16321646.

(47) Tomic, S.; Sunderland, A. G.; Bush, I. J. Parallel Multi-Band k.p Code for Electronic Structure of Zinc Blend Semiconductor Quantum Dots. J. Mater. Chem. 2006, 16, 1963-1972.

(48) Aeberhard, U.; Vaxenburg, R.; Lifshitz, E.; Tomic, S. Fluorescence of Colloidal $\mathrm{PbSe} / \mathrm{PbS}$ QDs in NIR Luminescent Solar Concentrators. Phys. Chem. Chem. Phys. 2012, 14, 16223-16228.

(49) Clark, S. J.; Segall, M. D.; Pickard, C. J.; Hasnip, P. J.; Probert, M. I. J.; Refson, K.; Payne, M. C. First Principles Methods Using CASTEP. Z. Kristallogr. - Cryst. Mater. 2005, 220, 567-570.

(50) Marques, M. A. L.; Oliveira, M. J. T.; Burnus, T. Libxc: A Library of Exchange and Correlation Functionals for Density Functional Theory. Comput. Phys. Commun. 2012, 183, 2272-2281.

(51) Tran, F.; Blaha, P. Accurate Band Gaps of Semiconductors and Insulators with a Semilocal Exchange-Correlation Potential. Phys. Rev. Lett. 2009, 102, 226401.

(52) An, J. M.; Franceschetti, A.; Dudiy, S. V.; Zunger, A. The Peculiar Electronic Structure of PbSe Quantum Dots. Nano Lett. 2006, 6, 2728-2735.

(53) Liljeroth, P.; Zeijlmans van Emmichoven, P. A.; Hickey, S. G.; Weller, H.; Grandidier, B.; Allan, G.; Vanmaekelbergh, D. Density of States Measured by Scanning-Tunneling Spectroscopy Sheds New Light on the Optical Transitions in PbSe Nanocrystals. Phys. Rev. Lett. 2005, 95, 086801.

(54) Li, X.-Q.; Nakayama, H.; Arakawa, Y. Phonon Bottleneck in Quantum Dots: Role of Lifetime of the Confined Optical Phonons. Phys. Rev. B: Condens. Matter Mater. Phys. 1999, 59, 5069-5073.

(55) Srivastava, G. P. The Physics of Phonons; Taylor \& Francis: New York, 1990

(56) Barman, S.; Srivastava, G. P. Lifetime of Nonequilibrium ZoneCenter Longitudinal Optical Phonons in Zinc-Blende Materials. Appl. Phys. Lett. 2002, 81, 3395-3397.

(57) Delaire, O.; Ma, J.; Marty, K.; May, A. F.; McGuire, M. A.; Du, M. H.; Singh, D. J.; Podlesnyak, A.; Ehlers, G.; Lumsden, M. D.; Sales, B. C. Giant Anharmonic Phonon Scattering in PbTe. Nat. Mater. 2011, 10, 614-619.

(58) Bartnik, A. C.; Efros, A. L.; Koh, W. K.; Murray, C. B.; Wise, F. W. Electronic States and Optical Properties of PbSe Nanorods and Nanowires. Phys. Rev. B: Condens. Matter Mater. Phys. 2010, 82, 195313.

(59) Zhang, Y.; Ke, X.; Chen, C.; Yang, J.; Kent, P. R. C. Thermodynamic Properties of $\mathrm{PbTe}, \mathrm{PbSe}$ and $\mathrm{PbS}$ : First-Principles Study. Phys. Rev. B: Condens. Matter Mater. Phys. 2009, 80, 024304. 